\title{
A generalization of Hull and White formula and applications to option pricing approximation
}

\author{
Elisa Alòs \\ Universitat Pompeu Fabra \\ c/Ramon Trias Fargas, 25-27 \\ 08005 Barcelona, Spain
}

February 4, 2004

\begin{abstract}
By means of Malliavin Calculus we see that the classical Hull and White formula for option pricing can be extended to the case where the noise driving the volatility process is correlated with the noise driving the stock prices. This extension will allow us to construct option pricing approximation formulas. Numerical examples are presented.

Keywords: continuous-time option pricing model, stochastic volatility, Malliavin Calculus.

JEL code: G130
\end{abstract}

\section{Introduction}

The work of Black and Scholes (1973) and Merton (1973) assumes that the stock prices $S_{t}$ satisfy a stochastic differential equation of the form

$$
d S_{t}=\mu S_{t} d t+\sigma S_{t} d W_{t}
$$

where $\mu$ and $\sigma$ are constants and $W$ is a standard Brownian motion. The parameter $\sigma$ is called the volatility of the model.

One of the main properties of this model is that it allows us to evaluate derivative prices by the use of simple analytical formulas. For example, the price of a call option with initial log stock price $x$, strike price $K$ and time to maturity $T-t$ is given by the function

$$
C_{B S}(t, x ; \sigma):=e^{x} N\left(d_{1}\right)-K e^{-r(T-t)} N\left(d_{2}\right),
$$

where $N$ denotes de standard normal distribution function, $r$ is the interest rate (that it is assumed to be constant) and

$$
d_{1,2}:=\frac{x-\ln K+\left(r \pm \frac{1}{2} \sigma^{2}\right)(T-t)}{\sigma \sqrt{T-t}}
$$


It is widely recognized that the simplicity of this popular model is not longer sufficient to capture modern market phenomena. In particular, the constant volatility assumption is clearly not true from empirical studies. One of the natural extensions of the Black-Scholes model that have been proposed in the literature and in practice is to modify the specification of volatility to make it a stochastic process. Some examples of modelling are: Hull and White (1987), Stein and Stein (1991), Ball and Roma (1994) and Heston (1993). However, new difficulties arise from this approach. In particular, these models are more complex, and then it is more difficult to obtain analytic formulas for option prices. Even when closed-form pricing solutions can be derived, the analytical computations are usually hard.

The simplest models for the volatility process assume that the volatility and the noise $W_{t}$ are uncorrelated (see for example Hull and White (1987), Stein and Stein (1991) and Ball and Roma (1994)). In this case, option prices are given by the so-called Hull and White formula:

$$
E^{*}\left(C_{B S}\left(t, x: \sqrt{\frac{1}{T-t} \int_{t}^{T} \sigma_{s}^{2} d s}\right) \mid \mathcal{F}_{t}\right),
$$

where $\left(\mathcal{F}_{t}\right)$ denotes the $\sigma$-algebra generated by the volatility process and $E^{*}$ denotes the expectation with respect to the risk-neutral probability (see for example Fouque, Papanicolau and Sircar (2000), Chapter 2). Nevertheless, it is often found from financial data that there exists a negative correlation between these two processes, and there are economic arguments that justify this negative correlation. But the correlated case is more difficult to handle mathematically, and the problem of deriving analytical formulas for option prices becomes more complex . Heston (1993) presented the first closed-form option pricing solution for a correlated model. Recently, closed-forms for other models have been obtained (see for example Bakshi, Cao and Chen (1997), Bates (1996), Bakshi and Chen (1997), Scott (1997) and Schöbel and Zhu (1999)).

As an alternative to this closed-form solutions, approximate option prices have been constructed. Fouque, Papanicolau and Sircar (2000) and Fouque, Papanicolau, Sircar and Solna (2003) presented a method to construct approximate option pricing formulas for the case of fast-mean reverting volatilities. The basic idea is to work in large intervals, where we can assume that the mean reversion is fast and then the constant-volatility model (with a correction to account for random volatility) is a good approximation. This results have been extended recently by Alòs (2003) to the case of general volatility models, where by means of Itô's lemma the derivative price is decomposed as the sum of four terms, which identify the main features of the market that affect to option prices: the expected future volatility, the correlation between the volatility and the noise driving the stock prices, the market price of volatility risk and the quadratic variation of the expected volatility process.

The main goal of this paper is to generalize the Hull and White formula to the correlated case. More precisely, we will see that the price of an european option can be decomposed as 
- the same derivative price if there where no correlation and

- a correction due by correlation.

This result can be interpreted as a generalization of Hull and White formula and will allow us to construct approximate option pricing formulas, similar to the presented by Alòs (2003), but simpler. The main technique we use in order to find this decomposition is the Itô's formula for anticipating processes. The anticipating stochastic calculus (or Malliavin Calculus ) is a powerfull extension of classical Itô's calculus that allows us to work with non-adapted processes (we refer to Nualart (1995) for a complete introduction to this subject). For this reason it reveals as a natural tool for our problem, where the average future volatility $\frac{1}{T-t} \int_{t}^{T} \sigma_{s}^{2} d s$ is not an adapted process.

This paper is organized as follows. In Section 2 we give a brief exposition of option pricing with equivalent measures. Section 3 is devoted to introduce some preliminaries on Malliavin Calculus. In Section 4 we prove the decomposition formulas for option prices and in Section 5 we use this decomposition in order to derive our approximation formula. Finally, in Section 6 we apply this formula to the extended Stein and Stein model presented in Schöbel and Zhu (1999). Some numerical results are presented, and we compare our approximated results with the exact values computed by Schöbel and Zhu (1999).

\section{Preliminaries on option pricing}

We will consider the following model for stock prices in a time interval $[0, T]$ :

$$
d S_{t}=\mu S_{t} d t+\sigma_{t} S_{t} d W_{t}, t \in[0, T]
$$

where $\mu$ is a constant, $W_{t}$ is a standard Brownian motion defined in a probability space $(\Omega, \mathcal{F}, P)$ and $\sigma_{t}$ is a square integrable process in $[0, T] \times \Omega$. We will assume that both $W_{t}$ and $\sigma_{t}$ adapted to the some filtration that we will denote

by $\left(\mathcal{F}_{t}\right)_{t \in[0, T]}$. It will be convenient in the following sections to make the change of variable

$$
X_{t}=\log \left(S_{t}\right), t \in[0, T]
$$

An European option is a contract that pays at maturity time $T$ a nonnegative payoff function $h\left(X_{T}\right)$ of he $\log$ stock price $X_{T}$ at time $T$. As particular examples we can consider:

- An European call option: gives its holder the right, but not the obligation, to buy one unit of an underlying asset for a predetermined strike price $K$ on the maturity time $T$. The asset is assumed not to pay dividends and ther are not transaction costs. In this case the payoff function is given by $h\left(X_{T}\right)=\left(e^{X_{T}}-K\right)_{+}$. 
- An European put option: gives its holder the right, but not the obligation, to sell one unit of the asset for a predetermined strike price $K$ on the maturity time $T$. Then $h\left(X_{T}\right)=\left(K-e^{X_{T}}\right)_{+}$.

At time $t<T$ this kind of contracts hve a value, known as the derivative price $V_{t}$, which will vary with $t$ and the observed stock prices until time $t$.

in the next subsection we present the general methodology for option pricing based in the Girsanov transformation. We will denote by $r$ the interest rate, that we will assume constant.

\subsection{Pricing with equivalent measures}

Suppose that there exists a probability distribution $P^{*}$ equivalent to the original one $P$ under which the discounted stock price process $\tilde{S}_{t}=e^{-r t} S_{t}$ is a martingale. It is well-known that if we price an European call by the formula

$$
V_{t}=e^{-r(T-t)} E^{*}\left[h\left(X_{T}\right) \mid \mathcal{F}_{t}\right],
$$

where $E^{*}$ denotes the expectation with respect to $P^{*}$, there is no arbitrage oportunity. Thus $V_{t}$ is a possible price for this derivative.

Let us now construct equivalent martingale measures. As the process $\tilde{S}_{t}$ satisfies the equation

$$
d \tilde{S}_{t}=(\mu-r) \tilde{S}_{t} d t+\sigma_{t} \tilde{S}_{t} d W_{t}
$$

we need, in order to absorb the drift term of $\tilde{S}_{t}$ in is martingale term, to set

$$
W_{t}^{*}=W_{t}+\int_{0}^{t} \frac{(\mu-r)}{\sigma_{s}} d s .
$$

On the other hand, if we assume that $\sigma_{t}$ depend on a second independent Brownian motion $Z$, any transformation of the form

$$
Z_{t}^{*}=Z_{t}+\int_{0}^{t} \gamma_{s} d s
$$

where $\gamma_{s}$ is a process such that the integral $\int_{0}^{t} \gamma_{s} d s$ is well defined, will not change the drift of $\tilde{X}_{t}$. By Girsanov's theorem we know that, if $\frac{(\mu-r)}{\sigma_{s}}$ and $\gamma_{s}$ are adapted and bounded processes there exists a probability distribution $P^{*}$ equivalent to the original one under which $W_{t}^{*}$ and $Z_{t}^{*}$ are independent standard Browian motions. Notice that any alloable choice of $\gamma$ leads then to an equivalent martingale measure and to a different no arbitrage price. This process $\gamma$ is called the risk premium factor or the market price of volatility risk.

Much research has investigated the range of possible prices in general settings. The approach that we will follows here is the same as used by Fouque, Papanicolau and Sircar (2000), where it is assumed that the market selects a unique equivalent martingale measure under which derivative contracts are priced. Notice that the value of the market's price of volatility risk $\gamma$ can be seen only in derivative prices, since $\gamma$ does not feature in the real world for the stock price. 


\section{Preliminaries on Malliavin Calculus}

Let us consider a standard Bronwian motion $W=\left\{W_{t}, t \in[0, T]\right\}$ defined in a complete probability space $(\Omega, \mathcal{F}, P)$.We will assume that $W$ is adapted with respecto to some filtration $\left(\mathcal{F}_{t}\right)_{t \in[0, T]}$. Set $H=L^{2}([0, T], \mathcal{B}([0, T]), \mu)$, where $\mu$ denotes the Lebesgue measure on $[0, T]$ and denote $W(h)=\int_{0}^{T} h(s) d W_{s}$ the Wiener integral of a deterministic function $h \in H$. Notice that $E\left(W(h) W\left(h^{\prime}\right)\right)=$ $\left\langle h, h^{\prime}\right\rangle_{H}$.

In the sequel we introduce the basic notation and results of the stochastic calculus of variations with respect to $W$. For a complete exposition we refer to Nualart (1995).

Let $\mathcal{S}$ be the set of smooth and cylindrical random variables of the form

$$
F=f\left(W\left(h_{1}\right), \ldots, W\left(h_{n}\right)\right),
$$

where $n \geq 1, f \in \mathcal{C}_{b}^{\infty}\left(\mathbb{R}^{n}\right)$ ( $f$ and all its derivatives are bounded), and $h_{1}, . ., h_{n} \in$ $H$. Given a random variable $F$ of the form, we define its derivative with respect to the Brownian motion $W$ as the stochastic process $\left\{D_{t}^{W} F, t \in[0, T]\right\}$ given by

$$
D_{t}^{W} F=\sum_{i=1}^{n} \frac{\partial f}{\partial x_{i}}\left(W\left(h_{1}\right), \ldots, W\left(h_{n}\right)\right) h_{i}(x), t \in[0, T] .
$$

The operator $D^{W}$ is a closable unbounded operator from $L^{2}(\Omega)$ into $L^{2}([0, T] \times \Omega)$. We denote by $\mathbb{D}^{1,2}$ the clousure of $\mathcal{S}$ with respect to the norm defined by

$$
\|F\|_{1,2}^{2}=\|F\|_{L^{2}(\Omega)}^{2}+\left\|D^{W} F\right\|_{L^{2}([0, T] \times \Omega)}^{2} .
$$

More generally, we can define the iterated derivative of a random variable in $\mathcal{S}$ by

$$
D_{t_{1}, \ldots, t_{n}}^{W, n} F=D_{t_{1}}^{W} \cdots D_{t_{n}}^{W} F
$$

The iterated derivative operator $D^{W, n}$ is a closable unbounded operator from $L^{2}(\Omega)$ into $L^{2}\left([0, T]^{n} \times \Omega\right)$. We denote by $\mathbb{D}^{n, 2}$ the clousure of $\mathcal{S}$ with respect to the norm defined by

$$
\|F\|_{n, 2}^{2}=\|F\|_{L^{2}(\Omega)}^{2}+\sum_{k=1}^{n}\left\|D^{W, k} F\right\|_{L^{2}\left([0, T]^{k} \times \Omega\right)}^{2} .
$$

We denote by $\delta^{W}$ the adjoint of the derivative operator $D^{W}$. That is, the domain of $\delta^{W}\left(\right.$ denoted by $\left.\operatorname{Dom} \delta^{W}\right)$ is the set of elements $u \in L^{2}([0, T] \times \Omega)$ such that there exists a constant $c$ satisfying

$$
\left|E \int_{0}^{T} D_{t}^{W} F u_{t} d t\right| \leq c\|F\|_{L^{2}(\Omega)},
$$


for all $F \in \mathcal{S}$. If $u \in \operatorname{Dom} \delta^{W}, \delta^{W}(u)$ is the element of $L^{2}(\Omega)$ characterized by

$$
E\left(\delta^{W}(u) F\right)=E \int_{0}^{T} D_{t}^{W} F u_{t} d t, F \in \mathcal{S} .
$$

The operator $\delta$ is an extension of the Itô integral (see Skorohod (1975)), in the sense that the set $L_{a}^{2}([0, T] \times \Omega)$ of square integrable and adapted processes is included in $\operatorname{Dom} \delta$ and the operator $\delta$ restricted to $L_{a}^{2}([0, T] \times \Omega)$ coincides with the Itô stochastic integral). We will make use of the notation $\delta^{W}(u)=\int_{0}^{T} u_{t} d W_{t}$ for any $u \in \operatorname{Dom} \delta^{W}$.

We recall that $\mathbb{L}^{n, 2}:=L^{2}\left([0, T] ; \mathbb{D}^{n, 2}\right)$ is included in the domain of $\delta$ for all $n \geq 1$, and for a process $u \in \mathbb{L}^{1,2}$ we can compute the variance of the Skorohod integral of $u$ as follows:

$$
E\left(\delta^{W}(u)\right)^{2}=E \int_{0}^{T} u_{t}^{2} d t+E \int_{0}^{T} \int_{0}^{T} D_{s}^{W} u_{t} D_{t}^{W} u_{s} d t d s .
$$

We will need the following results on the Skorohod integral (see Nualart (1995).

Proposition 1 Let $u \in D o m \delta^{W} \delta$ and consider a random variable $F \in \mathbb{D}^{1,2}$ such that $E\left(F^{2} \int_{0}^{T} u_{t}^{2} d t\right)<\infty$. Then

$$
\int_{0}^{T} F u_{t} d W_{t}=F \int_{0}^{T} u_{t} d W_{t}-\int_{0}^{T}\left(D_{t} F\right) u_{t} d t
$$

in the sense that $F u \in D o m \delta$ if and only if the right-hand side of (4) is square integrable.

Proposition 2 Let $u \in L_{a}^{2}([0, t] \times \Omega)$. Then, for all $0 \leq t \leq s \leq T$

$$
D_{s}^{W} u_{t}=0 .
$$

\subsection{Itô's formula}

Here we will prove the following version of Itô's formula for anticipating processes.

Theorem 3 Let us consider a process of the form $X_{t}=X_{0}+\int_{0}^{t} u_{s} d W_{s}+\int_{0}^{t} v_{s} d s$, where $X_{0}$ is a $\mathcal{F}_{0}$-measurable random variable and $u, v \in L_{a}^{2}([0, T] \times \Omega)$. Consider also a process $Y_{t}=\int_{t}^{T} \theta_{s} d s$, for some $\theta \in \mathbb{L}^{1,2}$. Let $F: \mathbb{R}^{3} \rightarrow \mathbb{R}$ a twice continuously differentiable function such that there exists a positive constant $C$ such that, for all $t \in[0, T], F$ and its derivatives evaluated in $\left(t, X_{t}, Y_{t}\right)$ are 
bounded by $C$. Then it follows that

$$
\begin{aligned}
F\left(t, X_{t}, Y_{t}\right)= & F\left(0, X_{0}, Y_{0}\right)+\int_{0}^{t} \frac{\partial F}{\partial s}\left(s, X_{s}, Y_{s}\right) d s \\
& +\int_{0}^{t} \frac{\partial F}{\partial x}\left(s, X_{s}, Y_{s}\right) d X_{s}+\int_{0}^{t} \frac{\partial F}{\partial y}\left(s, X_{s}, Y_{s}\right) d Y_{s} \\
& +\int_{0}^{t} \frac{\partial^{2} F}{\partial x \partial y}\left(s, X_{s}, Y_{s}\right)\left(D^{-} Y\right)_{s} u_{s} d s \\
& +\frac{1}{2} \int_{0}^{t} \frac{\partial^{2} F}{\partial x^{2}}\left(s, X_{s}, Y_{s}\right) u_{s}^{2} d s,
\end{aligned}
$$

where $\left(D^{-} Y\right)_{s}:=\int_{s}^{T} D_{s}^{W} Y_{r} d r$.

Proof. This proof is similar to the proof of Theorem 3 in Alòs and Nualart (1998). Here we will give only a skech of this proof. Fix $n \geq 1$ and take $t_{i}=i t / n$. Applying Taylor development up to the second order we obtain that

$$
\begin{aligned}
F\left(t, X_{t}, Y_{t}\right)= & F\left(0, X_{0}, Y_{0}\right)+\sum_{i=0}^{n} \frac{\partial F}{\partial t}\left(t_{i}, X_{t_{i}}, Y_{t_{i}}\right)\left(t_{i+1}-t_{i}\right) \\
& +\sum_{i=0}^{n} \frac{\partial F}{\partial x}\left(t_{i}, X_{t_{i}}, Y_{t_{i}}\right)\left(X_{t_{i+1}}-X_{t_{i}}\right) \\
& +\sum_{i=0}^{n} \frac{\partial F}{\partial y}\left(t_{i}, X_{t_{i}}, Y_{t_{i}}\right)\left(Y_{t_{i+1}}-Y_{t_{i}}\right) \\
& +\sum_{i=0}^{n} \frac{\partial^{2} F}{\partial t^{2}}\left(\bar{t}_{i}, \bar{X}_{t_{i}}, \bar{Y}_{t_{i}}\right)\left(t_{i+1}-t_{i}\right)^{2} \\
& +\sum_{i=0}^{n} \frac{\partial^{2} F}{\partial t \partial x}\left(\bar{t}_{i}, \bar{X}_{t_{i}}, \bar{Y}_{t_{i}}\right)\left(t_{i+1}-t_{i}\right)\left(X_{t_{i+1}}-X_{t_{i}}\right) \\
& +\sum_{i=0}^{n} \frac{\partial^{2} F}{\partial t \partial y}\left(\bar{t}_{i}, \bar{X}_{t_{i}}, \bar{Y}_{t_{i}}\right)\left(t_{i+1}-t_{i}\right)\left(Y_{t_{i+1}}-Y_{t_{i}}\right) \\
& +\sum_{i=0}^{n} \frac{\partial^{2} F}{\partial x^{2}}\left(\bar{t}_{i}, \bar{X}_{t_{i}}, \bar{Y}_{t_{i}}\right)\left(X_{t_{i+1}}-X_{t_{i}}\right)^{2} \\
& +\sum_{i=0}^{n} \frac{\partial^{2} F}{\partial x \partial y}\left(\bar{t}_{i}, \bar{X}_{t_{i}}, \bar{Y}_{t_{i}}\right)\left(X_{t_{i+1}}-X_{t_{i}}\right)\left(Y_{t_{i+1}}-Y_{t_{i}}\right) \\
& +\sum_{i=0}^{n} \frac{\partial^{2} F}{\partial y^{2}}\left(\bar{t}_{i}, \bar{X}_{t_{i}}, \bar{Y}_{t_{i}}\right)\left(Y_{t_{i+1}}-Y_{t_{i}}\right)^{2} \\
= & F\left(0, X_{0}, Y_{0}\right) \\
& +T_{1}+T_{2}+T_{3}+T_{4}+T_{5}+T_{6}+T_{7}+T_{8}+T_{9} .
\end{aligned}
$$

for some intermediate point $\left(\bar{t}_{i}, \bar{X}_{t_{i}}, \bar{Y}_{t_{i}}\right)$ between $\left(t_{i}, X_{t_{i}}, Y_{t_{i}}\right)$ and $\left(t_{i+1}, X_{t_{i+1}}, Y_{t_{i+1}}\right)$. Now the proof will be decomposed into several steps. 
Step 1. It is clear, by classical arguments, that

$$
T_{1} \rightarrow \int_{0}^{t} \frac{\partial F}{\partial s}\left(s, X_{s}, Y_{s}\right) d s
$$

and

$$
T_{3} \rightarrow \int_{0}^{t} \frac{\partial F}{\partial y}\left(s, X_{s}, Y_{s}\right) \theta_{s} d s
$$

in $L^{1}(\Omega)$.

Step 2. We can write

$$
\begin{aligned}
T_{2}= & \sum_{i=0}^{n} \frac{\partial F}{\partial x}\left(t_{i}, X_{t_{i}}, Y_{t_{i}}\right)\left(\int_{t_{i}}^{t_{i+1}} v_{s} d s\right) \\
& +\sum_{i=0}^{n} \frac{\partial F}{\partial x}\left(t_{i}, X_{t_{i}}, Y_{t_{i}}\right)\left(\int_{t_{i}}^{t_{i+1}} u_{s} d W_{s}\right) .
\end{aligned}
$$

Again from classical arguments, it is easy to see that

$$
\sum_{i=0}^{n} \frac{\partial F}{\partial x}\left(t_{i}, X_{t_{i}}, Y_{t_{i}}\right)\left(\int_{t_{i}}^{t_{i+1}} v_{s} d s\right) \underset{L^{1}(\Omega)}{\rightarrow} \int_{0}^{t} \frac{\partial F}{\partial x}\left(s, X_{s}, Y_{s}\right) v_{s} d s .
$$

On the other hand, by Proposition 1 we it follows that

$$
\begin{aligned}
& \sum_{i=0}^{n} \frac{\partial F}{\partial x}\left(t_{i}, X_{t_{i}}, Y_{t_{i}}\right)\left(\int_{t_{i}}^{t_{i+1}} u_{s} d W_{s}\right) \\
= & \sum_{i=0}^{n} \int_{t_{i}}^{t_{i+1}} \frac{\partial F}{\partial x}\left(t_{i}, X_{t_{i}}, Y_{t_{i}}\right) u_{s} d W_{s} \\
& +\sum_{i=0}^{n} \int_{t_{i}}^{t_{i+1}} D_{s}^{W}\left(\frac{\partial F}{\partial x}\left(t_{i}, X_{t_{i}}, Y_{t_{i}}\right)\right) u_{s} d s
\end{aligned}
$$

By the chain rule for the derivative operator (see Nualart (1995) and Proposition 2 we deduce that

$$
\int_{t_{i}}^{t_{i+1}} D_{s}^{W}\left(\frac{\partial F}{\partial x}\left(t_{i}, X_{t_{i}}, Y_{t_{i}}\right)\right) u_{s} d s=\int_{t_{i}}^{t_{i+1}} \frac{\partial^{2} F}{\partial x \partial y}\left(t_{i}, X_{t_{i}}, Y_{t_{i}}\right)\left(D_{s}^{W} Y_{t_{i}}\right) u_{s} d s .
$$

Now, by the same arguments as in Alòs and Nualart ( 1998) we can see that

$$
\sum_{i=0}^{n} \int_{t_{i}}^{t_{i+1}} \frac{\partial^{2} F}{\partial x \partial y}\left(t_{i}, X_{t_{i}}, Y_{t_{i}}\right)\left(D_{s}^{W} Y_{t_{i}}\right) u_{s} \underset{L^{1}(\Omega)}{\longrightarrow} \int_{0}^{t} \frac{\partial^{2} F}{\partial x \partial y}\left(s, X_{s}, Y_{s}\right)\left(D^{-} Y\right)_{s} u_{s} d s .
$$


Step 4. Using again the same arguments as in Alòs and Nualart ( 1998) we can see that

$$
T_{7} \underset{L^{1}(\Omega)}{\longrightarrow} \frac{1}{2} \int_{0}^{t} \frac{\partial^{2} F}{\partial x^{2}}\left(s, X_{s}, Y_{s}\right) u_{s}^{2} d s
$$

Step 5. From the facts that $F$ and its derivatives are bounded, $\int_{0}^{T} E v_{s}^{2} d s<$ $\infty$ and $\int_{0}^{T} E \theta_{s}^{2} d s$ it follows that $T_{4}+T_{5}+T_{6}+T_{8}+T_{9}$ tends to zero in $L^{1}(\Omega)$.

Step 6 . Finally, we will prove that for all $t \in[0, T]$, the process

$$
\frac{\partial F}{\partial x}\left(t_{i}, X_{t_{i}}, Y_{t_{i}}\right) u_{s} \mathbf{1}_{[0, t]}(s)
$$

belongs to the domain of $\delta$ and that

$$
\begin{aligned}
& \int_{0}^{t} \frac{\partial F}{\partial x}\left(s, X_{s}, Y_{s}\right) u_{s} d W_{s} \\
= & F\left(t, X_{t}, Y_{t}\right)-F\left(0, X_{0}, Y_{0}\right)-\int_{0}^{t} \frac{\partial F}{\partial s}\left(s, X_{s}, Y_{s}\right) d s \\
& -\int_{0}^{t} \frac{\partial F}{\partial x}\left(s, X_{s}, Y_{s}\right) v_{s} d s-\int_{0}^{t} \frac{\partial F}{\partial y}\left(s, X_{s}, Y_{s}\right) d Y_{s} \\
& -\int_{0}^{t} \frac{\partial^{2} F}{\partial x \partial y}\left(s, X_{s}, Y_{s}\right)\left(D^{-} Y\right)_{s} u_{s} d s \\
& -\frac{1}{2} \int_{0}^{t} \frac{\partial^{2} F}{\partial x^{2}}\left(s, X_{s}, Y_{s}\right) u_{s}^{2} d s .
\end{aligned}
$$

In order to obtain this result we will apply Lemma 1 in Alòs and Nualart (1998) to the sequence of processes

$$
\Phi_{s}^{n}=u_{s} \sum_{i=0}^{2^{n}-1} \frac{\partial F}{\partial x}\left(t_{i}, X_{t_{i}}, Y_{t_{i}}\right) \mathbf{1}_{\left[t_{i}, t_{i+1}\right]}(s) .
$$

We have that $\Phi^{n}$ converges in $L^{2}([0, T] \times \Omega)$ to $\Phi$ as $n$ teds to infinity. From the previous steps we obtain that

$$
\delta\left(\Phi^{n}\right)=\sum_{i=0}^{n} \int_{t_{i}}^{t_{i+1}} \frac{\partial F}{\partial x}\left(t_{i}, X_{t_{i}}, Y_{t_{i}}\right) u_{s} d W_{s}
$$

converges in $L^{1}(\Omega)$ to a random variable equal to the right-hand side of Equation (6). Then, in order to complete the proof it suffices to prove that the right-hand side of Equation (6) belongs to $L^{2}(\Omega)$. This follows easily from the hypotheses of the theorem.

\section{An extension of Hull and White formula}

Consider the stock prices model (1) presented in Section 2. We will also use the following notation: 
- $v_{t}^{2}=\frac{1}{T-t} \int_{t}^{T} E^{*}\left(\sigma_{s}^{2} \mid \mathcal{F}_{t}\right) d s$. That is, $v_{t}^{2}$ denotes the expected average volatility under the risk-neutral probability $P^{*}$.

- $C_{B S}(t, x ; \sigma)$ will denote the price of a call option for a model with constant volatility equal to $\sigma$, current $\log$ stock price $x$, time to maturity $T-t$, strike price $K$ and interest rate $r$. That is (see for example Lamberton and Lapeyre (1991), Section 3.2):

$$
C_{B S}(t, x ; \sigma)=e^{x} N\left(d_{1}\right)-K e^{-r(T-t)} N\left(d_{2}\right),
$$

where

$$
d_{1,2}:=\frac{x-\log K+\left(r \pm \frac{\sigma^{2}}{2}\right)(T-t)}{\sigma \sqrt{T-t}}
$$

and

$$
N(d)=\frac{1}{\sqrt{2 \pi}} \int_{-\infty}^{d} e^{-y^{2} / 2} d y
$$

- $\mathcal{L}_{B S}(\sigma)$ will denote the Black-Scholes differential operator (in the log variable) with volatility $\sigma$ :

$$
\mathcal{L}_{B S}(\sigma)=\frac{\partial}{\partial t}+\frac{1}{2} \sigma^{2} \frac{\partial^{2}}{\partial x^{2}}+\left(r-\frac{1}{2} \sigma^{2}\right) \frac{\partial}{\partial x}-r .
$$

It is well known that $\mathcal{L}_{B S}(\sigma) C_{B S}(\cdot, \cdot ; \sigma)=0$.

We will consider also the following hypotheses:

(H1) There exist a positive real constant $a$ such that $a \leq \nu_{t}^{2}$ for all $t \in[0, T]$.

(H2) $\sigma^{2} \in \mathbb{L}^{1,2}([0, T])$

(H3) For all $t \in[0, T]$ there exists a positive and $\mathcal{F}_{t}$-measurable random variable $A_{t}$ such that for all $s \in[t, T]$,

$$
\left|E^{*}\left(\left(\int_{s}^{T} D_{s}^{W} \sigma_{r}^{2} d r\right) \sigma_{s} \mid \mathcal{F}_{t}\right)\right| \leq A_{t},
$$

where $E^{*}$ denotes the expectation with respect to the risk-neutral probability $P^{*}$.

We will make also use of the following lemma, similar to Lemma 5 in Fouque, Papanicolau, Sircar and Solna (1993)

Lemma 4 Assume that Hypothesis (H1) holds. Then, for all $n \geq 2$ and $0 \leq$ $t \leq s \leq T$

$$
\begin{aligned}
& E^{*}\left(\left|\frac{\partial^{n} C_{B S}}{\partial x^{n}}\left(s, X_{s}, \nu_{s}\right)\right| \mid \sigma_{u}, u \in[t, s]\right) \\
\leq & C(T-s)^{1-n / 2},
\end{aligned}
$$

for some positive constant $C$. 
Proof. An easy calculation gives us that

$$
\frac{\partial C_{B S}}{\partial x}(s, x, \sigma)=e^{x} N\left(d_{1}\right),
$$

from where we deduce that, for all $n \geq 2$

$$
\begin{aligned}
\frac{\partial^{n} C_{B S}}{\partial x^{n}}(s, x, \sigma) & =e^{x} \sum_{i=0}^{n}\left(\begin{array}{c}
n \\
i
\end{array}\right) \frac{\partial^{i}}{\partial x^{i}}\left(N\left(d_{1}\right)\right) \\
& =e^{x} \sum_{i=0}^{n-1}\left(\begin{array}{l}
n \\
i
\end{array}\right) \frac{N^{(i)}\left(d_{1}\right)}{\sigma^{i} \sqrt[i]{T-s}} .
\end{aligned}
$$

As

$$
N^{\prime}\left(d_{1}\right)=\frac{e^{-d_{1}^{2}}}{2 \sqrt{\pi}}
$$

it is easy to see that, for all $i \geq 1$

$$
N^{(i)}\left(d_{1}\right)=N^{\prime}\left(d_{1}\right) \sum_{j=1}^{n-1} c_{j} d_{1}^{j},
$$

for some positive constants $c_{j}$. Notice that for all $a<1$

$$
e^{-\theta^{2}} \theta^{2}=e^{-a \theta^{2}} e^{-(1-a) \theta^{2}} \theta^{2} \leq k e^{-a \theta^{2}},
$$

for some $k>0$. Then

$$
N^{(i)}\left(d_{1}\right) \leq c e^{-c^{\prime} d_{1}^{2}}
$$

for some positive constants $c$ and $c^{\prime}$, which implies that

$$
\left|\frac{\partial^{n} C_{B S}}{\partial x^{n}}(s, x, \sigma)\right| \leq \frac{c e^{x} e^{-c^{\prime} d_{1}^{2}}}{(\sigma \sqrt{T-s})^{n-1}} .
$$

Then we can write

$$
\begin{aligned}
& E^{*}\left(\left|\frac{\partial^{n} C_{B S}}{\partial x^{n}}\left(s, X_{s}, \nu_{s}\right)\right| \mid \sigma_{u}, u \in[t, s]\right) \\
\leq & \frac{c}{(\sqrt{T-s})^{n-1}} E^{*}\left(e^{X_{s}} \exp \left(-c^{\prime}\left(\frac{X_{s}-\log K}{\nu_{s} \sqrt{T-t}}\right)^{2}\right) \mid \sigma_{u}, u \in[t, s]\right) .
\end{aligned}
$$

Denote

$$
u=x-\ln K
$$

and

$$
\tau=\nu_{s} \sqrt{T-t} .
$$


Then expression (8) can be rewritten as

$$
\begin{aligned}
& \frac{c K}{(T-s)^{\frac{n-1}{2}}} \int_{\mathbb{R}} e^{u} e^{-c^{\prime}\left(\frac{u}{\tau}\right)^{2} / 2} p(u) d u \\
= & \frac{c K}{(T-s)^{\frac{n}{2}-1}} \int_{\mathbb{R}} e^{\tau u} e^{-c^{\prime} u^{2} / 2} p(\tau u) d u,
\end{aligned}
$$

where $p$ denotes the conditional expectation of $u \equiv X_{s}-\ln K$.This allows us to complete the proof.

We will need to use the following corollary of this result.

Corollary 5 Consider a process $\sigma=\left\{\sigma_{t}, t \in[0, T]\right\}$ satisfying hypotheses (H1), (H2) and (H3). Then, for all $n \geq 1$ and for all $0 \leq t \leq T$

$$
\begin{aligned}
E^{*} & \left(\int_{t}^{T} e^{-r(s-t)}\left(\frac{\partial^{3}}{\partial x^{3}}-\frac{\partial^{2}}{\partial x^{2}}\right) C_{B S}\left(s, X_{s}, v_{s}\right)\right. \\
& \left.\times\left(\int_{s}^{T} D_{s}^{W} \sigma_{r}^{2} d r\right) \sigma_{s} d s \mid \mathcal{F}_{t}\right) \\
\leq & C A_{t}(T-t)^{\frac{1}{2}} .
\end{aligned}
$$

Proof. In fact, using Lemma 4 and hypotheses (H1), (H2) and (H3) we can write

$$
\begin{aligned}
& E^{*}\left(\int_{t}^{T} e^{-r(s-t)}\left(\frac{\partial^{3}}{\partial x^{3}}-\frac{\partial^{2}}{\partial x^{2}}\right) C_{B S}\left(s, X_{s}, v_{s}\right)\right. \\
& \left.\times\left(\int_{s}^{T} D_{s}^{W} \sigma_{r}^{2} d r\right) \sigma_{s} d s \mid \mathcal{F}_{t}\right) \\
= & E^{*}\left(\int_{t}^{T} e^{-r(s-t)} E^{*}\left[\left(\frac{\partial^{3}}{\partial x^{3}}-\frac{\partial^{2}}{\partial x^{2}}\right) C_{B S}\left(s, X_{s}, v_{s}\right) \mid \sigma_{u}, u \in[t, s]\right]\right. \\
& \left.\times\left(\int_{s}^{T} D_{s}^{W} \sigma_{r}^{2} d r\right) \sigma_{s} d s \mid \mathcal{F}_{t}\right) \\
\leq & C \int_{t}^{T} e^{-r(s-t)}(T-s)^{1-\frac{3}{2}} E^{*}\left(\left(\int_{s}^{T} D_{s}^{W} \sigma_{r}^{2} d r\right) \sigma_{s} \mid \mathcal{F}_{t}\right) d s \\
\leq & C^{\prime} A_{t} .
\end{aligned}
$$

as we wanted to prove.

Now we are in a position to prove the main result of this paper.

Theorem 6 (Decomposition formula) Assume the model (1), where

$$
\sigma=\left\{\sigma_{s}, s \in[0, T]\right\}
$$


is an adapted and square integrable process such that hypotheses (H1), (H2) and (H3) hold. Then, for all $t \in[0, T]$

$$
\begin{aligned}
& V_{t}=E^{*}\left(C_{B S}\left(t, X_{t} ; v_{t}\right) \mid \mathcal{F}_{t}\right) \\
& +\frac{1}{2} E^{*}\left(\int_{t}^{T} e^{-r(s-t)}\left(\frac{\partial^{3}}{\partial x^{3}}-\frac{\partial^{2}}{\partial x^{2}}\right) C_{B S}\left(s, X_{s}, v_{s}\right)\right. \\
& \left.\quad \times\left(\int_{s}^{T} D_{s}^{W} \sigma_{r}^{2} d r\right) \sigma_{s} d s \mid \mathcal{F}_{t}\right)
\end{aligned}
$$

Proof. Notice that $C_{B S}\left(T, X_{T} ; v_{T}\right)=V_{T}$. As $e^{-r t} V_{t}$ is a $P^{*}$-martingale we can write

$$
e^{-r t} V_{t}=E^{*}\left(e^{-r T} V_{T} \mid \mathcal{F}_{t}\right)=E^{*}\left(e^{-r T} C_{B S}\left(T, X_{T} ; v_{T}\right) \mid \mathcal{F}_{t}\right) .
$$

Now our idea is to apply Itô's formula (5) to the process $e^{-r t} C_{B S}\left(t, X_{t} ; v_{t}\right)$. As the derivatives of $C_{B S}(t, x ; y)$ are not bounded we will make use of an approximating argument. Take $\delta>0$ and consider the process

$$
e^{-r t} C_{B S}\left(t-\delta, X_{t} ; v_{t}^{\delta}\right),
$$

where $v_{t}^{\delta}:=\sqrt{\frac{1}{T-t+\delta} \int_{t}^{T+\delta} \sigma_{s}^{2} d s}$. Applying Itô's formula (5) with $Y_{t}=\int_{t}^{T+\delta} \sigma_{\theta}^{2} d \theta$ (notice that $v_{t}^{\delta}:=\sqrt{\frac{1}{T-t+\delta} Y_{t}}$ ) we deduce that

$$
\begin{aligned}
& e^{-r T} C_{B S}\left(T-\delta, X_{T} ; v_{T}^{\delta}\right) \\
= & e^{-r t} C_{B S}\left(t-\delta, X_{t} ; v_{t}^{\delta}\right) \\
& +\int_{t}^{T} e^{-r s}\left(\mathcal{L}_{B S}\left(v_{s}\right)+\frac{1}{2}\left(\sigma_{s}^{2}-\left(v_{s}^{\delta}\right)^{2}\right)\left(\frac{\partial^{2}}{\partial x^{2}}-\frac{\partial}{\partial x}\right)\right) \\
& \quad \times C_{B S}\left(s-\delta, X_{s}, v_{s}^{\delta}\right) d s \\
& +\int_{t}^{T} e^{-r s}\left(\frac{\partial C_{B S}}{\partial x}\right)\left(s-\delta, X_{s}, v_{s}^{\delta}\right) \sigma_{s} d W_{s}^{*} \\
& +\frac{1}{2} \int_{t}^{T} e^{-r s}\left(\frac{\partial^{2} C_{B S}}{\partial x \partial \sigma}\right)\left(s-\delta, X_{s}, v_{s}^{\delta}\right) \frac{\left(D_{s}^{-} Y_{s}\right) \sigma_{s}}{v_{s}^{\delta}(T-s+\delta)} d s \\
& -\frac{1}{2} \int_{t}^{T} e^{-r s} \frac{\partial C_{B S}}{\partial \sigma}\left(s-\delta, X_{s}, v_{s}^{\delta}\right) \frac{\left(\sigma_{s}^{2}-\left(v_{s}^{\delta}\right)^{2}\right)}{v_{s}^{\delta}(T-s+\delta)} d s,
\end{aligned}
$$

which implies that

$$
\begin{aligned}
& e^{-r T} C_{B S}\left(T-\delta, X_{T} ; v_{T}^{\delta}\right) \\
= & e^{-r t} C_{B S}\left(t-\delta, X_{t} ; v_{t}^{\delta}\right) \\
& +\int_{t}^{T} e^{-r s}\left(\frac{\partial C_{B S}}{\partial x}\right) C_{B S}\left(s-\delta, X_{s}, v_{s}^{\delta}\right) \sigma_{s} d W_{s}^{*} \\
& +\frac{1}{2} \int_{t}^{T} e^{-r s}\left(\frac{\partial^{2} C_{B S}}{\partial x \partial \sigma}\right)\left(s-\delta, X_{s}, v_{s}^{\delta}\right) \frac{\left(D_{s}^{-} Y_{s}\right) \sigma_{s}}{v_{s}^{\delta}(T-s+\delta)} d s
\end{aligned}
$$


Taking conditional expectations and multiplying by $e^{r t}$ we obtain that

$$
\begin{aligned}
& E^{*}\left[C_{B S}\left(T-\delta, X_{T} ; v_{T}^{\delta}\right) \mid \mathcal{F}_{t}\right] \\
= & C_{B S}\left(t-\delta, X_{t} ; v_{t}^{\delta}\right) \\
& +E^{*}\left(\int_{t}^{T} e^{-r(s-t)}\left(\frac{\partial^{2} C_{B S}}{\partial x \partial \sigma}\right)\left(s-\delta, X_{s}, v_{s}^{\delta}\right) \frac{\left(D_{s}^{-} Y_{s}\right) \sigma_{s}}{v_{s}^{\delta}(T-s+\delta)} \mid \mathcal{F}_{t}\right)
\end{aligned}
$$

Moreover, from the classical relationship (see for example Fouque, Papanicolau, Sircar and Solna (2003), pg. 8) between the Gamma (derivative with respect to the stock price) and the Vega (derivative with respect to the volatility) we deduce that

$$
\frac{\partial C_{B S}}{\partial \sigma}(s, x, \sigma) \frac{1}{\sigma(T-s)}=\left(\frac{\partial^{2}}{\partial x^{2}}-\frac{\partial}{\partial x}\right) C_{B S}(s, x, \sigma),
$$

which allows us to write

$$
\begin{aligned}
& E^{*}\left[C_{B S}\left(T-\delta, X_{T} ; v_{T}^{\delta}\right) \mid \mathcal{F}_{t}\right] \\
= & C_{B S}\left(t-\delta, X_{t} ; v_{t}^{\delta}\right) \\
& +\frac{1}{2} E^{*}\left(\int_{t}^{T} e^{-r(s-t)}\left(\frac{\partial^{3}}{\partial x^{3}}-\frac{\partial^{2}}{\partial x^{2}}\right) C_{B S}\left(s-\delta, X_{s}, v_{s}^{\delta}\right)\left(D_{s}^{-} Y_{s}\right) \sigma_{s} \mid \mathcal{F}_{t}\right) \\
= & C_{B S}\left(t-\delta, X_{t} ; v_{t}^{\delta}\right) \\
& +\frac{1}{2} E^{*}\left(\int_{t}^{T} e^{-r(s-t)}\left(\frac{\partial^{3}}{\partial x^{3}}-\frac{\partial^{2}}{\partial x^{2}}\right) C_{B S}\left(s-\delta, X_{s}, v_{s}^{\delta}\right)\right. \\
& \left.\quad \times\left(\int_{s}^{T} D_{s}^{W} \sigma_{r}^{2} d r\right) \sigma_{s} \mid \mathcal{F}_{t}\right)
\end{aligned}
$$

Letting now $\delta \rightarrow 0$ and using (H1), (H3), Corollary 5 and the dominated convergence theorem we obtain that

$$
\begin{aligned}
V_{t}= & E^{*}\left(C_{B S}\left(t, X_{t} ; v_{t}\right) \mid \mathcal{F}_{t}\right) \\
+\frac{1}{2} E^{*} & \left(\int_{t}^{T} e^{-r(s-t)}\left(\frac{\partial^{3}}{\partial x^{3}}-\frac{\partial^{2}}{\partial x^{2}}\right) C_{B S}\left(s, X_{s}, v_{s}\right)\right. \\
& \left.\times\left(\int_{s}^{T} D_{s}^{W} \sigma_{r}^{2} d r\right) \sigma_{s} d s \mid \mathcal{F}_{t}\right)
\end{aligned}
$$

and now the proof is complete.

\section{Option pricing approximation formulas}

In this section we will use the decomposition formula proved in the last section in order to construct approximate option pricing formulas. Let us introduce 
some preliminary notation in order to simplify the exposition. We will denote

$$
H\left(t, X_{t}, v_{t}\right)=\left(\frac{\partial^{3}}{\partial x^{3}}-\frac{\partial^{2}}{\partial x^{2}}\right) C_{B S}\left(t, X_{t}, v_{t}\right),
$$

and

$$
U_{t}=\int_{t}^{T}\left(\int_{s}^{T} D_{s}^{W} \sigma_{r}^{2} d r\right) \sigma_{s} d s .
$$

We remark that the decomposition formula can then be rewritten as

$$
V_{t}=E^{*}\left(C_{B S}\left(t, X_{t} ; v_{t}\right) \mid \mathcal{F}_{t}\right)+\frac{1}{2} E^{*}\left(\int_{t}^{T} e^{-r(s-t)} H\left(s, X_{s}, v_{s}\right) d U_{s} \mid \mathcal{F}_{t}\right)
$$

In order to prove our approximation result we need to introduce the following hypotheses:

(H4) $\sigma^{2} \in \mathbb{L}^{2,2}([0, T])$

(H5) For all $t \in[0, T]$ there exists a positive and $\mathcal{F}_{t}$-measurable random variable $A_{t}$ and some $\gamma>1$ such that for all $s \in[t, T]$,

$$
\left|E^{*}\left(U_{s} d U s \mid \mathcal{F}_{t}\right)\right| \leq A_{t}(T-s)^{\gamma},
$$

and

$$
\left|E^{*}\left(D_{s}^{-} U_{s} \mid \mathcal{F}_{t}\right)\right| \leq A_{t},
$$

where $E^{*}$ denotes the expectation with respect to the risk-neutral probability $P^{*}$

Theorem 7 (Price expansion) Assume the model (1), where $\sigma=\left\{\sigma_{s}, s \in[0, T]\right\}$ is an adapted and square integrable process such that hypotheses (H1) to (H5) hold. Then, for all $t \in[0, T]$

$$
\begin{aligned}
V_{t}= & E^{*}\left(C_{B S}\left(t, X_{t} ; v_{t}\right)+\frac{1}{2} H\left(t, X_{t}, v_{t}\right) U_{t} \mid \mathcal{F}_{t}\right) \\
& +E^{*}\left\{\frac{1}{4} \int_{t}^{T} e^{-r(s-t)}\left(\frac{\partial^{3}}{\partial x^{3}}-\frac{\partial^{2}}{\partial x^{2}}\right) H\left(s, X_{s}, v_{s}\right) U_{s} d U_{s} d s\right. \\
& \left.+\frac{1}{2} \int_{t}^{T} e^{-r(s-t)} H\left(s, X_{s}, v_{s}\right) D_{s}^{-} U_{s} d s \mid \mathcal{F}_{t}\right\}
\end{aligned}
$$

Proof. In Theorem 1 we have proved that

$$
\begin{aligned}
V_{t}= & E^{*}\left(C_{B S}\left(t, X_{t} ; v_{t}\right) \mid \mathcal{F}_{t}\right) \\
& +\frac{1}{2} E^{*}\left(\int_{t}^{T} e^{-r(s-t)} H\left(s, X_{s}, v_{s}\right) d U_{s} \mid \mathcal{F}_{t}\right)
\end{aligned}
$$


Consider now the process $e^{-r t} H\left(t, X_{t} ; v_{t}\right) U_{t}$. It is easy to check that

$$
e^{-r T} H\left(T, X_{T} ; v_{T}\right) U_{T}=0 .
$$

Then, by the same arguments as in the proof of Theorem 1 it follows that

$$
\begin{aligned}
0= & E^{*}\left\{H\left(t, X_{t} ; v_{t}\right) U_{t}\right. \\
& -\frac{1}{2} \int_{t}^{T} e^{-r(s-t)} H\left(s, X_{s}, v_{s}\right)\left(\int_{s}^{T} D_{s}^{W} \sigma_{r}^{2} d r\right) \sigma_{s} d s \\
& +\frac{1}{4} \int_{t}^{T} e^{-r(s-t)}\left(\frac{\partial^{3}}{\partial x^{3}}-\frac{\partial^{2}}{\partial x^{2}}\right) H\left(s, X_{s}, v_{s}\right) U_{s} d U_{s} \\
& \left.+\frac{1}{2} \int_{t}^{T} e^{-r(s-t)} H\left(s, X_{s}, v_{s}\right) D_{s}^{-} U_{s} d s \mid \mathcal{F}_{t}\right\},
\end{aligned}
$$

from where we deduce the desired result.

Remark 8 This result suggests that the quantity

$$
E^{*}\left(C_{B S}\left(t, X_{t} ; v_{t}\right)+\frac{1}{2} H\left(t, X_{t}, v_{t}\right) U_{t} \mid \mathcal{F}_{t}\right)
$$

can be shown as a 'first order approximation' to option prices.

Remark 9 As shown in Hull and White (1987) as well as in Ball and Roma (1994) or Alos (2003), the term

$$
E^{*}\left(C_{B S}\left(t, X_{t} ; v_{t}\right) \mid \mathcal{F}_{t}\right)
$$

(corresponding to the non-correlated case) can be approximated very accurately by replacing $v_{t}^{2}$ by $\left(E^{*} v_{t}^{2} \mid \mathcal{F}_{t}\right)$. In the same sense, the term $H\left(t, X_{t}, v_{t}\right)$ would be approximated in a natural way by

$$
H\left(t, X_{t}, \sqrt{E^{*}\left(v_{t}^{2} \mid \mathcal{F}_{t}\right)}\right) .
$$

This means that expression (11) can be approximated by

$$
\begin{aligned}
& V_{\text {aprox }}=C_{B S}\left(t, X_{t} ; \sqrt{E^{*}\left(v_{t}^{2} \mid \mathcal{F}_{t}\right)}\right) \\
& +\frac{1}{2} H\left(t, X_{t}, \sqrt{E^{*}\left(v_{t}^{2} \mid \mathcal{F}_{t}\right)}\right) E^{*}\left(U_{t} \mid \mathcal{F}_{t}\right) .
\end{aligned}
$$

Obviously the goodness of this approximation will depend on several factors: we will need the remaining term in Theorem 2

$$
\begin{aligned}
& +\frac{1}{4} \int_{t}^{T} e^{-r(s-t)}\left(\frac{\partial^{3}}{\partial x^{3}}-\frac{\partial^{2}}{\partial x^{2}}\right) H\left(s, X_{s}, v_{s}\right) U_{s} d U_{s} \\
& \left.+\frac{1}{2} \int_{t}^{T} e^{-r(s-t)} H\left(s, X_{s}, v_{s}\right) D_{s}^{-} U_{s} d s \mid \mathcal{F}_{t}\right\},
\end{aligned}
$$

to be small enought, as well as the variability of $v_{t}^{2}$. In order to see how to apply this formulas in practice, we will present an example in the next section. 


\section{An Example}

\subsection{The generalized Stein and Stein model}

In this section we consider that the volatility follows a mean-reverting OU process. As assumed by Stein and Stein (1991), the volatility process can be described, under the risk-neutralized probability, by

$$
d \sigma_{t}=-\alpha\left(m-\sigma_{t}\right) d t+c d B_{t},
$$

where $\alpha, m$ and $c$ are positive real constants and $B_{t}$ is a standard Brownian motion. Here we will assume, as in Schöbel and Zhu (1999), that $B_{t}=\rho B_{t}+$ $\sqrt{1-\rho^{2}} Z_{t}$, for some $\rho \in[-1,1]$ and for some standard Brownian motion $Z$ independent of $W$, which extends the S\&S classical model. Analytic formulas for option prices in this context have been obtained in Schöbel and Zhu (1999).

It is easy to see that for all $0 \leq t \leq s \leq T$,

$$
\sigma_{s}=m+\left(\sigma_{t}-m\right) e^{-\alpha(s-t)}+c \int_{t}^{s} e^{-\alpha(s-u)} d B_{u}
$$

From this expression it is clear that hypothesis (H1) does not hold. Nevertheless, for every $\varepsilon>0$ we can consider the process $\left(\sigma^{\varepsilon}\right)^{2}:=\varepsilon+\sigma^{2}$, which satisfies (H1). On the other hand, it is clear that $\sigma^{2}$ (and then $\left.\left(\sigma^{\varepsilon}\right)^{2}\right) \in \mathbb{L}^{1,2}$ (H2). Moreover, using the chain rule for the derivative operator (see Nualart (1995)) it follows that

$$
\begin{aligned}
D_{s}^{W}\left(\sigma_{r}^{\varepsilon}\right)^{2} & =D_{s}^{W} \sigma_{r}^{2} \\
& =2 \sigma_{r} D_{s} \sigma_{r} \\
& =2 c \rho \sigma_{r} e^{-\alpha(r-s)}
\end{aligned}
$$

from where we deduce that

$$
\begin{aligned}
& E^{*}\left(\left(\int_{s}^{T} D_{s}^{W} \sigma_{r}^{2} d r\right) \sigma_{s} \mid \mathcal{F}_{t}\right) \\
= & 2 c \rho \int_{s}^{T} e^{-\alpha(r-s)} E^{*}\left(\sigma_{r} \sigma_{s} \mid \mathcal{F}_{t}\right) d r \\
= & 2 c \rho\left\{M_{t}(s) \int_{s}^{T} e^{-\alpha(r-s)} M_{t}(r) d r\right. \\
& \left.+c^{2} \int_{s}^{T} e^{-\alpha(r-s)}\left(\int_{t}^{s} e^{-\alpha(s-u)} e^{-\alpha(r-u)}\right) d r\right\},
\end{aligned}
$$

which implies that hypothesis (H3) holds.

We can see also that $\sigma^{2}$ (and then $\left.\left(\sigma^{\varepsilon}\right)^{2}\right) \in \mathbb{L}^{2,2}(\mathrm{H} 4)$, and that

$$
\begin{aligned}
D_{\theta}^{W} D_{s}^{W}\left(\sigma_{r}^{\varepsilon}\right)^{2} & =D_{\theta}^{W} D_{s}^{W} \sigma_{r}^{2} \\
& =2 c \rho e^{-\alpha(r-s)} D_{\theta}^{W} \sigma_{r} \\
& =2 c^{2} \rho^{2} e^{-\alpha(r-s)} e^{-\alpha(r-\theta)} .
\end{aligned}
$$


Then we can deduce that

$$
\begin{aligned}
& E^{*}\left(U_{s} d U_{s} \mid \mathcal{F}_{t}\right) \\
= & E^{*}\left(\left(\int_{s}^{T}\left(\int_{r}^{T} D_{r}^{W} \sigma_{\theta}^{2} d \theta\right) \sigma_{r} d r\right)\left(\int_{s}^{T} D_{s}^{W} \sigma_{u}^{2} d u\right) \sigma_{s} \mid \mathcal{F}_{t}\right) \\
= & 4 c^{2} \rho^{2} E^{*}\left(\left(\int_{s}^{T} \sigma_{r} \int_{r}^{T} e^{-\alpha(\theta-r)} \sigma_{\theta} d \theta d r\right)\left(\int_{s}^{T} e^{-\alpha(u-s)} \sigma_{u} d u\right) \sigma_{s} \mid \mathcal{F}_{t}\right),
\end{aligned}
$$

and

$$
\begin{aligned}
E^{*}\left(D_{s}^{-} U_{s} \mid \mathcal{F}_{t}\right) & =E^{*}\left(\int_{s}^{T}\left(\int_{r}^{T} D_{s}^{W} D_{r}^{W} \sigma_{\theta}^{2} d \theta\right) \sigma_{r} d r \mid \mathcal{F}_{t}\right) \\
& =2 c^{2} \rho^{2} E^{*}\left(\int_{s}^{T}\left(\int_{r}^{T} e^{-\alpha(\theta-r)} e^{-\alpha(\theta-s)} d \theta\right) \sigma_{r} d r \mid \mathcal{F}_{t}\right),
\end{aligned}
$$

from where hypothesis (H5) follows. It the coefficients $c$ and $\rho$ as well as the time interval are small enought, it seems reasonable to assume that the remaining term

$$
\begin{aligned}
& +\frac{1}{4} \int_{t}^{T} e^{-r(s-t)}\left(\frac{\partial^{3}}{\partial x^{3}}-\frac{\partial^{2}}{\partial x^{2}}\right) H\left(s, X_{s}, v_{s}\right) U_{s} d U_{s} \\
& \left.+\frac{1}{2} \int_{t}^{T} e^{-r(s-t)} H\left(s, X_{s}, v_{s}\right) D_{s}^{-} U_{s} d s \mid \mathcal{F}_{t}\right\}
\end{aligned}
$$

as well as the variance of $\nu_{t}^{2}$ is small. Then we can try apply formula (12). The next Proposition is devoted to evaluate the quantities $E^{*}\left(v_{t}^{2} \mid \mathcal{F}_{t}\right)$ and $U_{t}$ for our particular volatility model.

Proposition 10 Consider the stock model (1), where, under the risk-neutral probability, the volatility process is given by the extended Stein and Stein model (13). Then

$$
E^{*}\left(v_{t}^{2} \mid \mathcal{F}_{t}\right)=\frac{1}{T-t} \int_{t}^{T}\left(M_{t}(s)+c^{2} F(s-t)\right) d s,
$$

and

$$
\begin{aligned}
& E^{*}\left(\int_{t}^{T}\left(\int_{s}^{T} D_{s}^{W} \sigma_{r}^{2} d r\right) \sigma_{s} d s \mid \mathcal{F}_{t}\right) \\
= & 2 c \rho\left(\int_{t}^{T} M_{t}(s) \int_{s}^{T} e^{-\alpha(r-s)} M_{t}(r) d r+c^{2} \int_{t}^{T} F(T-s) F(s) d s\right)
\end{aligned}
$$

where $M_{t}(s)=m+\left(\sigma_{t}-m\right) e^{-\alpha(s-t)}$ and $F(u)=\int_{0}^{u} e^{-2 \alpha \theta} d \theta$. 
Proof. We can write

$$
E^{*}\left(v_{t}^{2} \mid \mathcal{F}_{t}\right)=\frac{1}{T-t} \int_{t}^{T} E^{*}\left(\sigma_{s}^{2} \mid \mathcal{F}_{t}\right) d s .
$$

From (14) it follows easily that

$$
E^{*}\left(\sigma_{s}^{2} \mid \mathcal{F}_{t}\right)=\left(m+\left(\sigma_{t}-m\right) e^{-\alpha(s-t)}\right)^{2}+c^{2} \int_{t}^{s} e^{-2 \alpha(s-u)} d u,
$$

from where (15) follows. On the other hand,

$$
\begin{aligned}
& E^{*}\left(\int_{t}^{T}\left(\int_{s}^{T} D_{s}^{W} \sigma_{r}^{2} d r\right) \sigma_{s} d s \mid \mathcal{F}_{t}\right) \\
= & 2 c \rho \int_{t}^{T} \int_{s}^{T} e^{-\alpha(r-s)} E^{*}\left(\sigma_{r} \sigma_{s} \mid \mathcal{F}_{t}\right) d r d s \\
= & 2 c \rho\left\{\int_{t}^{T} M_{t}(s) \int_{s}^{T} e^{-\alpha(r-s)} M_{t}(r) d r d s\right. \\
& \left.+c^{2} \int_{t}^{T} \int_{s}^{T} e^{-\alpha(r-s)}\left(\int_{t}^{s} e^{-\alpha(s-u)} e^{-\alpha(r-u)}\right) d r\right\} \\
= & 2 c \rho\left\{\int_{t}^{T} M_{t}(s) \int_{s}^{T} e^{-\alpha(r-s)} M_{t}(r) d r\right. \\
& \left.+c^{2} \int_{t}^{T}\left(\int_{s}^{T} e^{-2 \alpha(r-s)} d r\right)\left(\int_{t}^{s} e^{-2 \alpha(s-u)} d u\right) d s\right\},
\end{aligned}
$$

which gives us (16). Now the proof is complete

Formula (12) and Proposition 1 give us a tool to approximate option prices. In the following tables we show some numerical applications of this approximation and we compare them with the exact values (into parentheses) calculated by analytical computations by Schöbel and Zhu (1999).

\begin{tabular}{|c|c|c|c|c|}
\hline$\rho \backslash K$ & 90 & 100 & 110 & 120 \\
\hline-1 & $15.444(15.416)$ & $8.338(8.307)$ & $3.503(3.468)$ & $1.033(0.995)$ \\
\hline-0.75 & $15.371(15.355)$ & $8.304(8.275)$ & $3.558(3.525)$ & $1.130(1.110)$ \\
\hline-0.5 & $15.298(15.292)$ & $8.270(8.243)$ & $3.613(3.582)$ & $1.228(1.218)$ \\
\hline-0.25 & $15.225(15.225)$ & $8.236(8.210)$ & $3.667(3.638)$ & $1.325(1.321)$ \\
\hline 0 & $15.152(15.155)$ & $8.203(8.176)$ & $3.722(3.694)$ & $1.422(1.420)$ \\
\hline 0.25 & $15.079(15.081)$ & $8.169(8.141)$ & $3.777(3.749)$ & $1.519(1.514)$ \\
\hline 0.5 & $15.006(15.003)$ & $8.135(8.106)$ & $3.832(3.803)$ & $1.617(1.605)$ \\
\hline 0.75 & $14.933(14.919)$ & $8.101(8.070)$ & $3.887(3.856)$ & $1.714(1.693)$ \\
\hline 1 & $14.860(14.828)$ & $8.067(8.034)$ & $3.942(3.909)$ & $1.811(1.777)$ \\
\hline
\end{tabular}

$$
X_{t}=\ln 100, r=0.0953, T=0.5, m=0.2, \sigma_{0}=0.2, \alpha=4, c=0.1
$$




\begin{tabular}{|c|c|c|c|c|}
\hline$\rho \backslash K$ & 90 & 100 & 110 & 120 \\
\hline-1 & $14.745(14.730)$ & $7.021(6.976)$ & $2.029(1.977)$ & $0.190(0.179)$ \\
\hline-0.75 & $14.684(14.679)$ & $6.973(6.936)$ & $2.092(2.051)$ & $0.275(0.279)$ \\
\hline-0.5 & $14.623(14.626)$ & $6.925(6.894)$ & $2.155(2.121)$ & $0.360(0.371)$ \\
\hline-0.25 & $14.562(14.576)$ & $6.877(6.849)$ & $2.219(2.189)$ & $0.445(0.458)$ \\
\hline 0 & $14.501(14.515)$ & $6.830(6.803)$ & $2.282(2.254)$ & $0.530(0.542)$ \\
\hline 0.25 & $14.441(14.456)$ & $6.781(6.753)$ & $2.345(2.316)$ & $0.615(0.621)$ \\
\hline 0.5 & $14.380(14.395)$ & $6.734(6.701)$ & $2.409(2.376)$ & $0.700(0.698)$ \\
\hline 0.75 & $14.319(14.330)$ & $6.686(6.645)$ & $2.472(2.433)$ & $0.784(0.773)$ \\
\hline 1 & $14.258(14.261)$ & $6.638(6.587)$ & $2.535(2.489)$ & $0.870(0.845)$ \\
\hline
\end{tabular}

$X_{t}=\ln 100, r=0.0953, T=0.5, m=0.1, \sigma_{0}=0.2, \alpha=4, c=0.1$

\begin{tabular}{|c|c|c|c|c|}
\hline$\rho \backslash K$ & 90 & 100 & 110 & 120 \\
\hline-1 & $16.383(16.357)$ & $9.800(9.777)$ & $5.110(5.084)$ & $2.268(2.235)$ \\
\hline-0.75 & $16.315(16.298)$ & $9.778(9.755)$ & $5.158(5.133)$ & $2.362(2.340)$ \\
\hline-0.5 & $16.247(16.236)$ & $9.756(9.732)$ & $5.207(5.182)$ & $2.457(2.441)$ \\
\hline-0.25 & $16.179(16.172)$ & $9.734(9.710)$ & $5.259(5.230)$ & $2.551(2.540)$ \\
\hline 0 & $16.111(16.172)$ & $9.711(9.687)$ & $5.305(5.279)$ & $2.646(2.635)$ \\
\hline 0.25 & $16.043(16.037)$ & $9.689(9.665)$ & $5.353(5.328)$ & $2.740(2.728)$ \\
\hline 0.5 & $15.975(15.964)$ & $9.667(9.642)$ & $5.402(5.377)$ & $2.834(2.819)$ \\
\hline 0.75 & $15.907(15.889)$ & $9.645(9.620)$ & $5.451(5.426)$ & $2.929(2.908)$ \\
\hline 1 & $15.840(15.810)$ & $9.623(9.598)$ & $5.499(5.475)$ & $3.023(2.994)$ \\
\hline
\end{tabular}

$X_{t}=\ln 100, r=0.0953, T=0.5, m=0.3, \sigma_{0}=0.2, \alpha=4, c=0.1$

\section{References}

[1] Alòs, E. (2003): A general decomposition formula for derivative prices in stochastic volatility models. Working Paper 665, Universitat Pompeu Fabra.

[2] Alòs, E. and Nualart, D. (1998): An extension of Itô's formula for anticipating processes. Journal of Theoretical Probability, 11 (2), 493-514.

[3] Ball, C. A. and Roma, A. (1994): Stochastic volatility option pricing. Journal of Financial and Quantitative Analysis 29, 589-607.

[4] Black, F. and Scholes, M. (1973): The pricing of options and corporate liabilities. Journal of Political Economy 3, 637-654.

[5] Comte, F. and Renault, E. (1998): Long-memory in continuous-time stochastic volatility models. Mathematical Finance 8, 291-323.

[6] Fouque, J-P., Papanicolau, G. and Sircar, K. R. (2000):Derivatives in Finantial markets with Stochastic Volatility. Cambridge. 
[7] Fouque, J-P., Papanicolau, G., Sircar, K. R. and Solna, K.(2003): Singular Perturbations in Option Pricing. Preprint.

[8] Heston, S. L. (1993): A closed-form solution for options with stochastic volatility with applications to bond and currency options. The Review of Finantial Studies 6, 327-343.

[9] Hu, Y. : Option princing in a market where the volatility is driven by fractional Brownian motions. Preprint.

[10] Hull, J. C. and White, A. (1987): The pricing of options on assets with stochastic volatilities. Journal of Finance 42, 281-300.

[11] Karatzas, I. and Shreve, S. E. (1991):Brownian motion and Stochastic Calculus. Springer-Verlag.

[12] Lamberton, D. and Lapeyre, B. (1991): Introduction au Calcul Stochastique Appliqué à la Finance. Ellipses-Edition Marketing.

[13] Merton, R. C. (1973): Theory of rational option pricing. Bell. J. Econom. and Management Sci. 4, 141-183.

[14] Nualart, D. (1995): The Malliavin Calculus and Related Topics. Springer.

[15] Stein, E. M. and Stein, J. C. (1991): Stock price distributions with stochastic volatility: an analytic approach. The review of Finantial Studies 4, 727-752. 